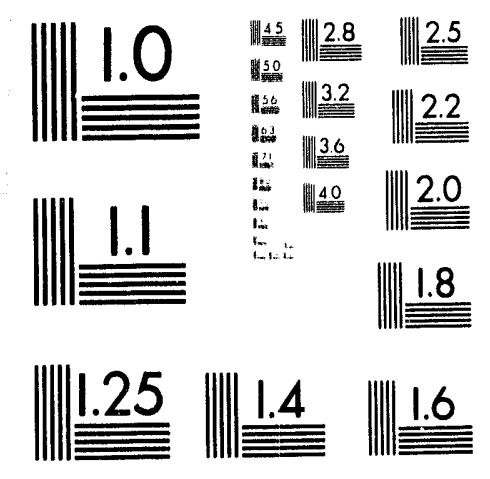



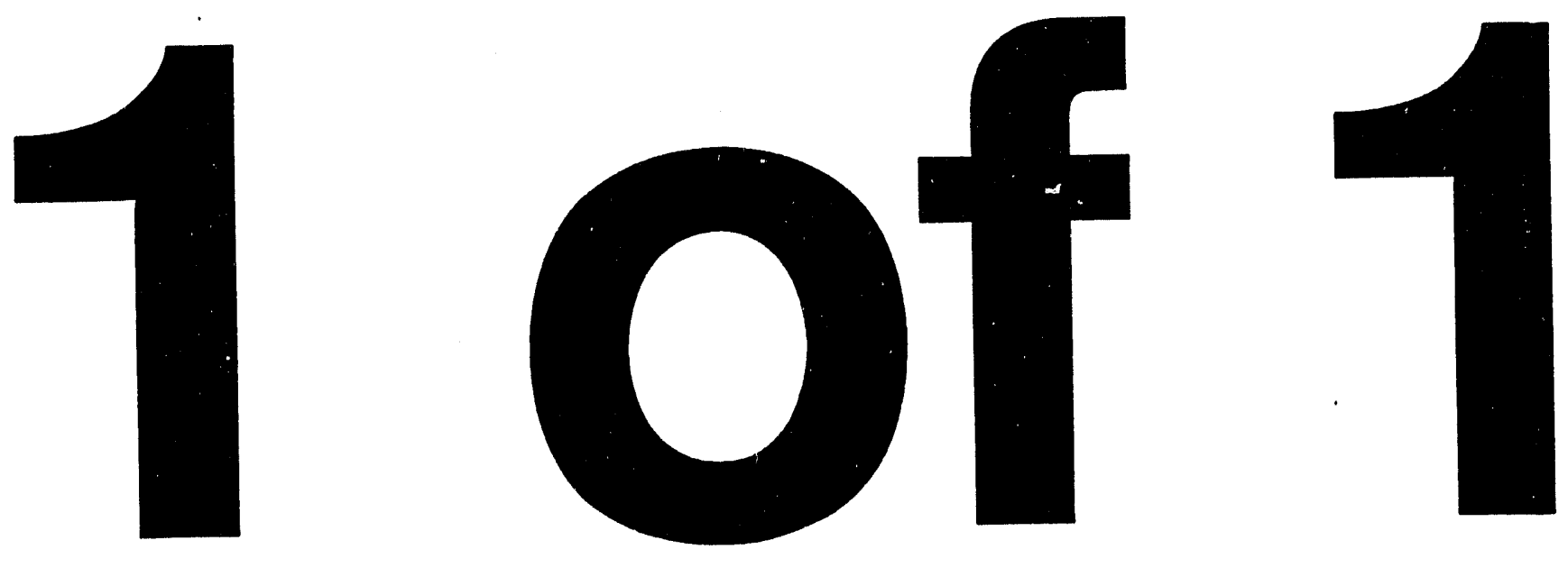


\title{
SUMMARY TALK ON BEAM CRYSTALLIZATION
}

\author{
J. P. Schiffer \\ Argonne National Laboratory, Argonne, IL 60439 U.S.A.
}

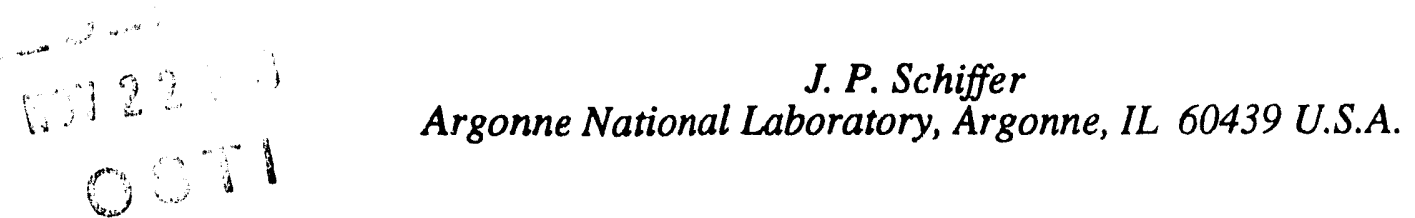

The logical ultimate limit of beam cooling leads to an ordered "crystalline" array of particles. It should occur when the thermal energy (not counting collective oscillations) becomes much less than the Coulomb energy stored between two neighboring particles in the beam:

$$
k T_{\text {incoh. }} \ll q^{2} / a
$$

where " $\mathrm{a}$ " is a parameter reflecting the interparticle spacing. As cooling techniques improve this limit is gradually coming within grasp. In fact, some think that it perhaps had already been reached in Novosibirsk [1] over a decade ago, but the evidence on this is not clear.

In this summary I would like to give a brief primer on what has been done in this area, indications from simulations of what one might expect in such a crystalline beam, and where some of the problems may be. I would like to fold in the contributions we heard and saw at this Workshop, give an assessment of where we now are, and of what the future may hold.

The idea and first simulations of a crystalline beam occurred around 1986 [2], and were then developed further and presented at the Wertheim Workshop in 1988 [3]. It was clear that the lowest energy configuration of particles, traveling in a focusing field that was cylindrically symmetric and constant in time, would be as is shown schematically in Fig. 1. The configuration depends on the linear density of particles. But since several small storage rings are similar in their size and focusing properties, we may here refer to these densities in terms of the approximate total number of particles $\mathrm{N}$ for a typical small storage ring. For $\mathrm{N}<10^{6}$ stored ions, the particles, when absolutely cold, would settle in a string. Then, as the density of particles increases so that the interparticle Coulomb

NL.P.21,227
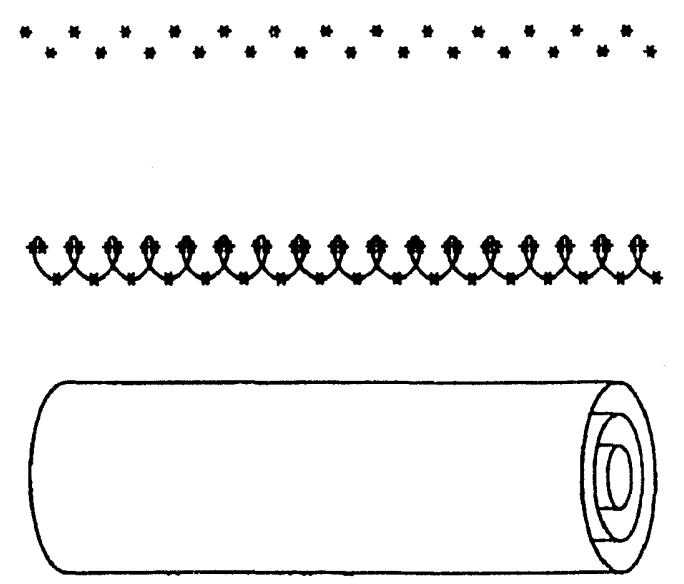

Fig. 1 Schematic representation of particles in a cold beam at various particle densities. The top view corresponds to one-dimensional order expected for $\mathrm{N}<10^{6}$, the next to a two-dimensional zig-zag, then to a helix, and at the bottom to multiple shells. forces became stronger than the focusing force, the particles would undergo dimensional phase transitions, first breaking out of a line into a twodimensional zig-zag pattern around $N=10^{6}$, then into various three-dimensional helical configurations on cylindrical surfaces above about $\mathrm{N} \approx 2 \times 10^{6}$, and for $\mathrm{N}$ $>10^{7}$ into several concentric shells. For the larger $N$ values these shells are equally spaced, with the surface density of particles on each shell the same, and thus the number of shells proportional to $\sqrt{\mathrm{N}}$.

But one has to deal with reality, both in the focusing method, and in considering the curvature inherent in a storage ring. What can one say about the best focusing scheme for a crystalline beam? One can obtain constant focusing in a weak-focusing betatron -- could this be the right method? At Wertheim some thought that this was a solution, most others thought otherwise, but at the present Workshop Ruggiero [4] has shown rigorously that weak focusing cannot work, strong focusing is absolutely needed for this ordered regime.

Can this ordering survive an alternating focusing field? At Wertheim, some simulations were discussed [3] that indicated that with the alternating field and with only longitudinal cooling, the early simulations assumed ideal cooling in both transverse and longitudinal directions. These simulations indicated that the beam did seem to remain in a multiple shell structure, very similar to the static case, but undergoing small shape oscillations. At the present Workshop Wei, Sessler and Li [5] presented 
simulations that included explicitly repeating FODO cells and bending, as well as vacuum chamber image charges that had not been considered in the earlier work. They also assumed cooling to constant angular velocity. Their systems for $\mathrm{N} \leqslant 10^{7}$ undergo simple oscillations and settle into a mode that requires no further cooling. Results were also shown [6] for larger $\left(\mathrm{N} \approx 10^{8}\right)$ systems that indicate no problems with alternating focusing, even if the longitudinal cooling is applied only once per orbit.

Next is the question of curvature. Since present beam cooling techniques force particles to travel with the same linear velocity, this introduces a problem when the particles are forced into a curved orbit with dipole elements. An ideal case was presented in the poster by Labrador, et al. [7] for a two-dimensional zig-zag pattern that was bent just the right amount for particles on the two strips of the zig-zag to move over by one spacing. This is a very special case. One would like to see a scheme in which the particles are "cooled" into a mode where they all travel with the same angular velocity, as was assumed in the contribution by Wei, et al. [5]. Note that this would imply a large coherent momentum spread across the beam, which could be as much as $\Delta \mathrm{p} / \mathrm{p}=10^{-4}$. Also, if the bending is not continuous, (continuous bending was assumed in Ref. 5) even a constant angular velocity beam would be subject to a longitudinal shear oscillation that may disrupt the order, particularly if individual elements bend by angles $\geq 60^{\circ}$.

For normal cooling to constant linear velocity, one may still wonder whether the interparticle forces within an ordered beam might be sufficient to resist shear. Simulations shown at Wertheim indicated that, not surprisingly, the amount of shear a given density of particles could resist depended on the ratio of the focusing force to the curvature of the storage ring, which is just the betatron tune. For the small storage rings, with betatron tunes around 2.5 , only the lowest density ordered beams would survive the shear: the one-dimensional string, which of course is not subject to shear, the zig-zag pattern and perhaps some of the simplest helical systems. The larger multi-shell systems would be too fragile and be disrupted by shear. Storage rings with large radii and large betatron tunes could sustain the more complex patterns of larger particle density because the shear would be smaller. The number of particles that can sustain bending should increase roughly as the 4th power of the betatron tune [6].

We need more realistic simulations however, that combine the periodic focusing with periodic bending, in order to better understand the limits at which cooling to fixed linear velocity might produce structures that can survive bending with conventional cooling schemes.

Where do we stand with results? At the time of Wertheim the only data in existence was the original Novosibirsk measurement. Since that time considerable progress has been made at a number of facilities, and at this Workshop very low temperatures have been reported both at the TSR and the ASTRID rings. We heard of the measurements at the TSR in Heidelberg from Habs [8], and they suggest that some sort of a limit may be approached at around $2 \times 10^{6}$ particles -- which is tantalizingly close to the limit to shear estimated earlier $[3,6]$ : a one- or twodimensional beam. It will be interesting to hear whether slight modifications in the tune of the ring modify this limiting number. Hangst [9] reported on measurements at Aarhus in ASTRID where low longitudinal temperatures, in much more dense beams of $\sim 10^{9}$ particles, show instabilities -- which conceivably could also be related to such problems.

Perhaps in these rings it would be best to focus on the low densities, $\mathrm{N}<10^{7}$ particles, as an initial goal. But at these low densities it is important to understand how long it takes to equilibrate longitudinal cooling with the perpendicular temperatures $T_{\perp}$. This may be a difficult issue to treat correctly in simulations [10] and the test against reality is going to happen soon.

Diagnostics are a major issue in this regime. We heard a paper by Avilov and Hofmann [11] on the Schottky spectra to be expected for one-dimensional beams -- but the relatively easy transition between one- and twodimensional configurations may complicate matters.

We heard about a possible new dedicated facility for crystalline beams from Tecchio: the CRYSTAL storage ring project at Legnaro [12]. This project has the potential of learning from and building on the detailed experiences at the existing small storage rings: the TSR and ASTRID, and thus optimizing their design for a new generation of specialized rings.

What does the future hold for this area? My guess is that within the next two years the evidence for ordering in one- and two-dimensional beams will become firmer at the storage rings with laser cooling. Beyond $\mathrm{N}=10^{7}, \mathrm{I}$ would guess that there will have to be some conceptual technical developments that would allow beams to attain constant angular velocity rather than constant linear velocity, before these forms of order may be realized in storage rings. But in the meantime many diagnostic questions need to be assessed. For instance, some thought and ideas are needed on how to separate the transverse beam temperature from coherent oscillations that are inherent to the beam and that do not disrupt order. And the consequences of the issue that was already mentioned, that the momentum spread in a crystalline beam must be quite appreciable -- though coherent, need to be considered.

Finally, one is confronted with the question: "What are crystalline beams good for?" I would like to answer this on three levels.

On the level of "basic science" these systems represent a unique form of condensed matter, that is some 15 orders of magnitude less dense than "normal" forms of matter in the solid state. Some of the features of such matter can also be studied in ion traps. But others are unique to beams. For instance, the one-dimensional Coulomb system 
never reaches complete order, but the distribution of spacings in such a system has unique mathematical interest. In random matrix theory, the Gaussian orthogonal ensemble [13], which is basic to discussions of the distribution of level spacings in atoms and nuclei and is a major aspect of quantum chaos, corresponds to the properties of a onedimensional Coulomb system at a particular temperature. Ott ar temperatures correspond to other statistical ensembles.

On the level of "applications" there are many aspects which may pertain to very cold beams that still remain to be investigated and understood. For instance, it is amusing to note that if two beams could be cooled to the spacecharge limit, and their crystalline structures could be aligned shell by shell to a micron or better, one might attain an improvement in luminosity by two orders of magnitude over the value for beams of the same diameter and no crystalline structure. Of course, even a slight misalignment would be catastrophic.

Finally on the human level, we always strive to try for the best, and it seems entirely appropriate that the limits of the "best" beam cooling be attempted, explored, and understood.

This research was supported by the U.S. Department of Energy, Nuclear Physics Division, under Contract W31-109-Eng-38.

\section{REFERENCES}

[1] E. N. Dementiev, et al., Sov. Phys. Tech. Phys. 25. 1001 (1980).

[2] J. P. Schiffer and P. Kienle, Z. Phys. A 321, 181 (1985); A. Rahman and J. P. Schiffer, Phys. Rev. Letts. 57, 1133 (1986); J. P. Schiffer and A. Rahman, Z.

Phys. A331, 71 (1988).

[3] J. P. Schiffer, Proc. of Workshop on Crystalline Ion Beams, Wertheim, FRG, Oct. 4-7, 1988, GSI-89-10 Report, April 1989, ISSN 0171-4546, p. 2.

[4] Alessandro Ruggiero, Demonstration of No Feasibility of a Crystalline Beam in a Betatron Magnet, Workshop on "Beam Cooling and Related Topics", October 3-8, 1993, Montreux, Switzerland.

[5] Jie Wei, Xiao-Ping Li, and Andrew M. Sessler, Critical Densities and Temperatures for Crystalline Beam, Workshop on "Beam Cooling and Related Topics", October 3-8, 1993, Montreux, Switzerland.

[6] J. P. Schiffer and J. S. Hangst, Molecular Dynamics Simulations of the Crystallization of Ion Beams in Alternating Focusing Fields, and for Curved Trajectories, Workshop on "Beam Cooling and Related Topics", October 3-8, 1993, Montreux, Switzerland.

[7] A. Labrador, S. Gustafsson, and A. Dainelli, Computer Simulation of a Crystalline Ion Beam in a Storage Ring, Workshop on "Beam Cooling and Related Topics", October 3-8, 1993, Montreux, Switzerland.

[8] H. J. Miesner, M. Grieser, R. Grimm, A. Gruber, D. Habs, W. Petrich, D. Schwalm, B. Wanner, H. Wernoe, Intrabeam Scattering Losses in Laser Cooling, Workshop on "Beam Cooling and Related Topics", October 3-8, 1993, Montreux, Switzerland.

[9] J. S. Hangst, J. S. Nielsen, O.Poulsen, J. P. Schiffer, P. Shi, and B. Wanner, Laser Cooling of a Bunched Beam in ASTRID, Workshop on "Beam Cooling and Related Topics", October 3-8, 1993, Montreux, Switzerland.

[10] J. P. Schiffer and J. S. Hangst, Z Phys. A 341, 107 (1991).

[11] V. V. Avilov and I. Hofmann, Theory of Longitudinal Schottky Spectra of Ordered Ion Beams in a Storage Ring, Workshop on "Beam Cooling and Related Topics", October 3-8, 1993, Montreux, Switzerland.

[12] L. Tecchio, G. Lamanna, V. Stagno, V. Variale, R. Calabrese, V. Guidi,F. Petrucci, G. Bisoffi, G. Ciullo, A. Dainelli, S. Gustafsson, A. Labrador, M. F. Moisio, A. Pisent, A. Ruggiero, and B. Yang, A Dedicated Storage Ring for Ion Beam Crystallization, Workshop on "Beam Cooling and Related Topics", October 3-8, 1993, Montreux, Switzerland.

[13] Freeman J. Dyson, J. Math. Phys. 3140 (1961).

\section{DISCLAIMER}

This report was prepared as an account of work sponsored by an agency of the United States Government. Neither the United States Government nor any agency thereof, nor any of their employees, makes any warranty, express or implied, or assumes any legal liability or responsibility for the accuracy, completeness, or usefulness of any information, apparatus, product, or process disclosed, or represents that its use would not infringe privately owned rights. Reference herein to any specific commercial product, process, or service by trade name, trademark, manufacturer, or otherwise does not necessarily constitute or imply its endorsement, recommendation, or favoring by the United States Government or any agency thereof. The views and opinions of authors expressed herein do not necessarily state or reflect those of the United States Government or any agency thereof. 

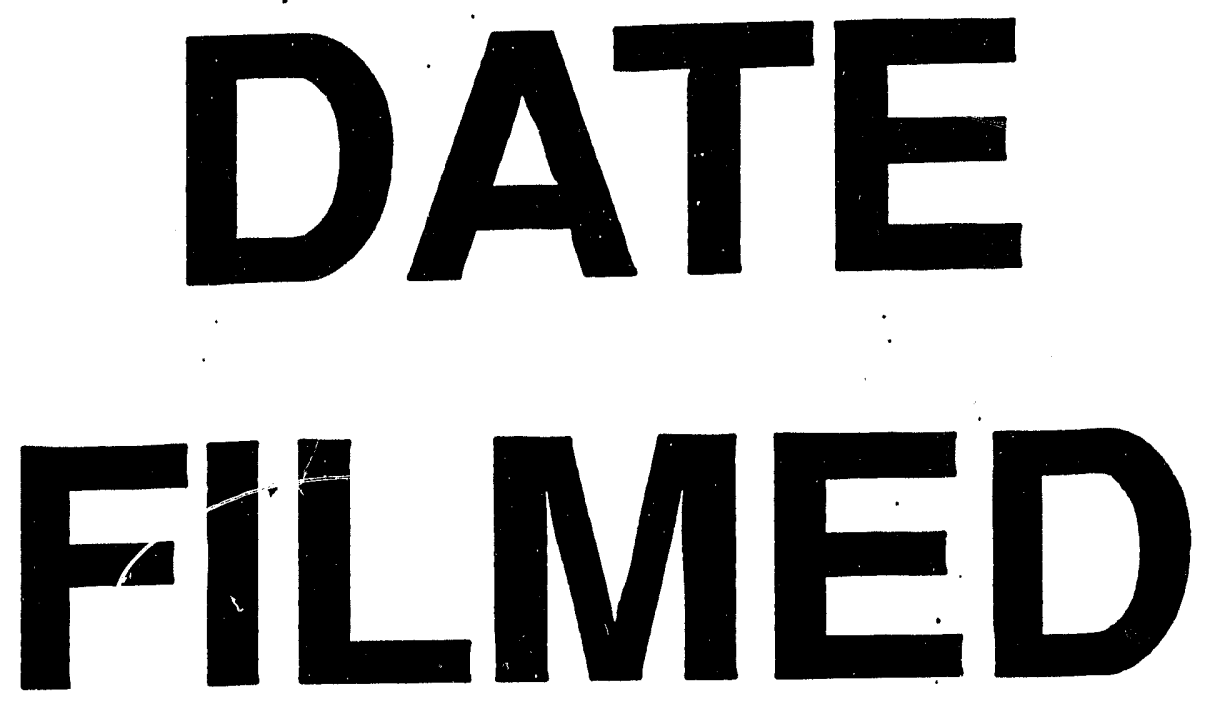

$12 / 29 / 93$
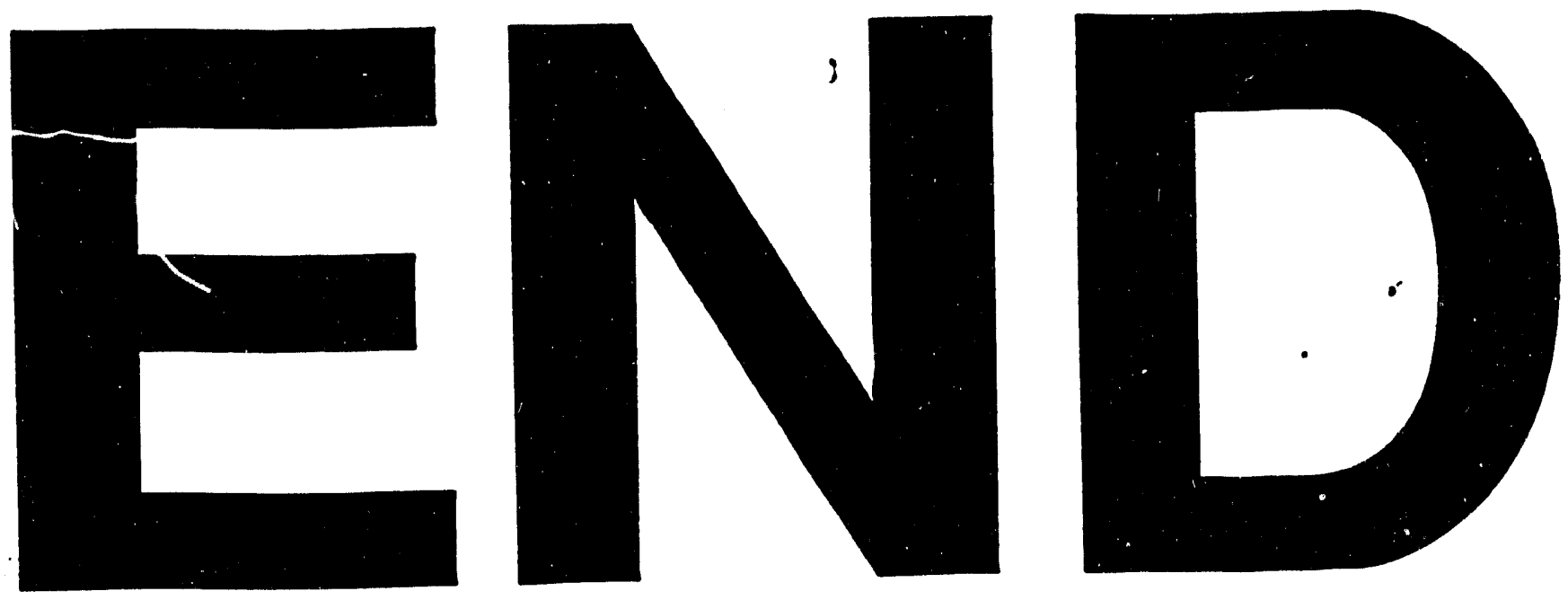
